Cahiers $d u \quad$ Cahiers du monde russe

MONDE RUSSE Russie - Empire russe - Union soviétique et États indépendants

$52 / 4 \mid 2011$

Varia

\title{
E. A. Rees, Iron Lazar
}

\section{Yves Cohen}

URL : http://journals.openedition.org/monderusse/7624

DOI : $10.4000 /$ monderusse. 7624

ISSN : $1777-5388$

\section{Éditeur}

Éditions de l'EHESS

\section{Édition imprimée}

Date de publication : 20 décembre 2011

Pagination : 746-750

ISBN : 978-2-7132-2353-2

ISSN : $1252-6576$

\section{Référence électronique}

Yves Cohen, «E. A. Rees, Iron Lazar », Cahiers du monde russe [En ligne], 52/4 | 2011, mis en ligne le 30 novembre 2012, Consulté le 25 septembre 2020. URL : http://journals.openedition.org/monderusse/ 7624 ; DOI : https://doi.org/10.4000/monderusse.7624

Ce document a été généré automatiquement le 25 septembre 2020

〔c École des hautes études en sciences sociales 


\section{E. A. Rees, Iron Lazar}

\section{Yves Cohen}

\section{RÉFÉRENCE}

E. A. REES, Iron Lazar. A Political Biography of Lazar Kaganovich. Londres - New York : Anthem Press, 2012, 373 pp.

1 Voici donc une solide biographie d'un personnage dont on savait toute l'importance dans la mise en place du stalinisme, puisqu'il a travaillé à partir de 1922 au plus près de Stalin à des postes de très grande responsabilité, avec toutefois une claire diminution de son influence à partir de la guerre, sinon déjà un peu avant. Jusqu'à présent, nous ne disposions que d'évocations comprises dans des récits sur Stalin, d'esquisses partielles liées en particulier à ses postes successifs de secrétaire du parti en Ukraine et à Moscou et de commissaire du peuple aux voies de communication et d'un insuffisant essai sur le commissaire de fer de Stalin. Il reste à savoir si cette biographie, aussi solide soit-elle, sera la dernière.

2 La question principale est de déterminer quel a été l'apport de Kaganovič dans la victoire de Stalin sur les autres dirigeants bolcheviks après la mort de Lenin puis dans l'établissement et la consolidation du pouvoir stalinien. Le livre est riche de ce point de vue. L'auteur est un historien confirmé du pouvoir stalinien qu'il a étudié précédemment sous bien des angles, à la fois directs et indirects, depuis l'histoire des dispositifs de contrôle de l'État et du parti, celle des chemins de fer, jusqu'à celle du culte et du pouvoir de Stalin lui-même. Dans le cas présent, il a consulté toutes les sources secondaires disponibles, toutes les sources publiées principalement depuis l'ouverture des archives à partir de la fin des années 1980 ainsi qu'un certain nombre de fonds d'archives, notamment en Ukraine. L'ouvrage fournit un nouvel éclairage sur ce personnage plus important en fin de compte que le «factotum » et «acolyte » de Stalin qu'il était aussi. Il ne laisse de côté aucune des inflexions des positions politiques de Kaganovič et de ses rapports avec les différents dirigeants. Il montre que ce vieux bolchevik, très brièvement proche de Trockij, s'est vite imposé à la fois comme un 
excellent organisateur et intellectuel organique du parti, capable de saisir et d'élaborer sa spécificité et de développer des formes d'organisation ajustées, et tout autant comme homme de terrain qui ne craint pas d'être directement confronté aux problèmes. Toutefois, plutôt que d'aborder l'action de Kaganovič sous l'angle pratique, Rees l'envisage plutôt sous l'angle politique. Or le livre montre que Kaganovič est un des artisans majeurs de la construction lente et profonde de ce pouvoir finement ramifié sur tout le territoire soviétique que Stalin édifie en six ans. Cela apparaît dès la guerre civile, Kaganovič est alors secrétaire de Nižnij-Novgorod: il se révèle à la fois un organisateur talentueux et un dirigeant impitoyable qui n'hésite pas à recourir aux exécutions d'otages pour asseoir le pouvoir soviétique dans un travail quotidien avec la Čéka. Il confirme ensuite ses aptitudes au Turkestan, puis en Ukraine, son pays de naissance, dont il est le secrétaire général du parti de 1925 à 1928 et, enfin, à l'échelle de toute l'Union soviétique, en se spécialisant dans les questions d'organisation et de normalisation du pays. Kaganovič est ainsi le penseur des "instructeurs" qui permettent au parti de contrôler les divers organes de gouvernement et donc les structures qui soutiennent la transformation du parti bolchevik de parti destiné à la prise du pouvoir en parti d'exercice du pouvoir dans une société non démocratique. Les instructeurs forment aussi une des armatures de base de l'Internationale communiste. L'activité et les articles de Kaganovič sont l'une des clés pour comprendre la mise en place efficace du rapport entre le parti et les soviets. Kaganovič est l'un de ceux pour lesquels la propension anti-démocratique du bolchevisme est absolument naturelle et sa complicité avec Stalin se construit en grande partie sur ce point. De même, Kaganovič justifie à plusieurs reprises le fait que l'État soviétique ne soit pas un État de droit et en fait un article de foi valable pour le monde et pour toute la trajectoire du communisme au pouvoir.

3 Cet ouvrage rend justice à un Kaganovič inventeur de nombreuses procédures ordinaires essentielles du pire pouvoir stalinien. Il est passé maître pour réduire l'initiative des organes inférieurs, centraliser, renforcer les contrôles, menacer, introduire la police politique dans les formes de la vie ordinaire au nom du bien prolétarien, le tout sur fond d'un discours parfaitement socialiste. Le moment venu, il signe les ordres d'exécution de masse, comme tous les autres membres du bureau politique. Dans le même temps, il affronte en Ukraine nombre de ses camarades en affirmant la nécessité de l'ukrainisation et, tout au long de sa carrière, il restera un agent actif de la promotion des cadres, procédé qui a permis au stalinisme de durer.

On sait qu'un des traits du pouvoir stalinien, qui ne dispose d'aucune forme de contrôle démocratique, consiste à envoyer depuis le centre des missi dominici pour mieux mettre en place des politiques de toutes sortes, en premier lieu les plus brutales, envers les populations et les tièdes du parti. Or c'est là ce qui convient le mieux à Kaganovič. Cet Ukrainien, cordonnier de métier, va au charbon. Le livre note tous ses déplacements plus ou moins prolongés sur les frontières en Ukraine, dans les kolkhozes de la collectivisation forcée, sur les chantiers du métro, sur les lieux de la famine galopante provoquée en 1932-1933 par sa propre politique et celle de ses amis, dans les régions pour y soutenir les méthodes de la Grande Terreur. Il n'y va pas seul, il est accompagné d'acolytes politiques et de policiers. Il y a en lui, qui a une puissance de travail phénoménale, un instrument majeur d'installation d'un pouvoir tout à la fois pour la conduite des opérations quotidiennes et pour la confrontation aux situations d'urgence. C'est au regard de cette particularité de Kaganovič si bien soulignée par Rees, que l'on déplore à cet ouvrage si érudit un manque littéralement stupéfiant : le 
très volumineux fonds personnel d'archives de Kaganovič, déclassifié depuis 1995 et complété depuis par de nombreux documents, n'est presque pas utilisé. On ne le trouve cité que vingt-cinq fois environ (citations tirées d'un moins grand nombre de documents encore) sur plus de 1600 notes.

5 Or ce fonds comporte des documents inestimables pour la compréhension de la pratique propre de Kaganovič. On le voit par exemple reporter de sa main les corrections elles-mêmes manuscrites apportées par Stalin à tel ou tel document, avant de le passer à la presse ou à d'autres instances du parti, pour que la part du maître ne soit connue de personne. Le fonds recèle aussi les agendas de Kaganovič sur de longues années. Ils sont certes d'un usage difficile qui nécessite une longue patience. En effet, les recharges amovibles ont été mélangées et les pages intercalées de façon passablement confuse. Ainsi, trois feuillets successifs portent la date du 26 septembre alors qu'ils relèvent manifestement chacun d'une année différente dans la décennie 1930. Mais, par exemple, même un survol aurait pu permettre de tirer profit des feuillets intitulés "Avec Stalin ", qui recueillent les points à discuter avec celui-ci. Il aurait également été possible de dater sans trop de difficulté tous ceux qui montrent Kaganovič, surtout en 1934, se rendre très souvent au siège de la GPU puis du NKVD : une invite à étudier de plus près son rôle sûrement primordial et encore peu connu dans le remplacement de l'un par l'autre et plus largement dans la gestion stalinienne directe de la police politique. D'autres documents montrent Kaganovič dans ses œuvres, de retour de mission pour organiser la terreur d'État avec des listes nominatives de cadres éliminés, et autant de lignes vides pour des postes désormais à pourvoir sans que les cadres locaux encore en place ne sachent plus à qui faire appel. Ils le montrent aussi, s'adressant au personnel du commissariat du peuple à l'industrie lourde en 1938, l'invitant à se reconstituer psychologiquement et à cesser de pleurnicher et ne trouvant rien de plus rassurant à dire que cette dénégation : « Nous extirpons les ennemis, nous ne les créons pas artificiellement » (RGASPI, 81/3/363/1-6, 25 janvier 1938). L'auteur de la prochaine biographie devra explorer ce fonds pour en extraire la substantifique moelle et nous conduire de façon approfondie dans l'étude plus détaillée des pratiques de gouvernement qui ont intrinsèquement produit ce pouvoir.

Il est d'autres points dont on attendait le traitement. Ainsi Kaganovič est-il réputé l'un des principaux inventeurs du vocabulaire du culte de Stalin. Quelques éléments déjà connus sont donnés mais la question n'est pas plus traitée que celle des pratiques de gouvernement. Comme elle, elle est indiquée, mais non pas l'état de l'art ni la possibilité éventuelle d'aller plus loin. L'ouvrage a privilégié une exposition chronologique alors qu'il aurait certainement gagné à introduire quelques chapitres thématiques, pour mieux renouveler l'histoire du stalinisme qui n'est pas seulement une histoire soviétique mais celle d'une dimension capitale, encore à interpréter plus profondément, du parcours du $\mathrm{Xx}^{\mathrm{e}}$ siècle. Des réponses sont bien tentées dans ce sens, mais elles restent peu satisfaisantes. L'une d'elles est l'usage que l'on peut faire dans l'enquête historique d'un diagnostic psychiatrique, celui bien sûr qui concerne Stalin vu comme psychopathe que le livre le reprend. En bonne méthode historienne, les actes des personnes, abordés par toutes les méthodes possibles, sont premiers par rapport à un diagnostic qui risque d'enfermer la richesse informative des sources dans une interprétation a priori. Obtient-on un véritable avantage interprétatif lorsqu'on avance, 
comme le fait l'ouvrage, que ce psychopathe réussi est parvenu à s'entourer de personnes saines?

7 L'ouvrage montre à foison comment Kaganovič est dans une soumission absolue au maître qu'il s'est choisi, ce qui n'était bien entendu pas ignoré. Mais on mesurait mal sa contribution personnelle, qui est différente de celle, plus administrative, de l'autre second complice majeur qu'est Molotov. Iron Lazar est un très bon exemple d'un genre indispensable, celui de l'histoire des seconds couteaux. Les seconds couteaux, comme Stalin l'a été avant de passer premier, sont les praticiens, ceux qui savent faire ce que les autres ne savent pas, en particulier grâce à leur talent d'organisation. Kaganovič est de ce point de vue d'une utilité cruciale pour Stalin. Mais il manque d'historiens des pratiques pour aller plus loin que ce livre dans cette direction.

Portrait exemplaire du stalinien, Kaganovič a constamment exposé le froid cynisme du politique appuyé sur la dynamique tellurique de l'histoire du monde qui va inexorablement vers le communisme et dont chacun doit se faire l'instrument implacable et impavide. De là, sans doute, qu'il soit mort en paix avec le monde et lui-même à 98 ans, quelques jours avant le putsch d'août 1991, à une adresse moscovite connue de tous depuis longtemps où il est à méditer que nulle victime ou parent de victime, pour autant qu'on le sache, ne soit venu lui demander des comptes en parole ou de manière plus physique. 